\title{
Abstract: Random-Forest-basierte Segmentierung der subkutanen Fettschicht der Mäusehaut in 3D-OCT-Bilddaten
}

\author{
Timo Kepp ${ }^{1}$, Christine Droigk ${ }^{1}$, Malte Casper ${ }^{2,3}$, Michael Evers ${ }^{2,3}$, \\ Nunciada Salma ${ }^{3}$, Dieter Manstein ${ }^{3}$, Heinz Handels ${ }^{1}$ \\ ${ }^{1}$ Institut für Medizinische Informatik, Universität zu Lübeck \\ ${ }^{2}$ Institut für Biomedizinische Optik, Universität zu Lübeck \\ ${ }^{3}$ Cutaneous Biology Research Center, Massachusetts General Hospital, Boston, MA \\ kepp@imi.uni-luebeck.de
}

Die Kryolipolyse ist ein nichtinvasives kosmetisches Verfahren zur lokalen Fettreduktion [1], bei der durch kontrollierte Kühlung selektiv subkutane Fettzellen zerstört werden. Für eine quantitative Evaluation des Verfahrens soll die subkutane Fettschicht in Mäusen segmentiert werden. Für eine Darstellung der Mäusehaut wurde die Optische Kohärenztomographie (OCT) als Bildmodalität genutzt, die eine detaillierte Aufnahme der subkutanen Fettschicht in Mikrometer-Auflösung ermöglicht. Aufgrund der großen Datenmenge ist eine manuelle Segmentierung der Daten nicht durchführbar. Daher präsentieren wir in diesem Beitrag einen Ansatz für die automatische Segmentierung der subkutanen Fettschicht der Mäusehaut in 3D-OCT-Bilddaten [2]. Hierzu verwenden wir einen Random Forest (RF), der auf manuell segmentierten B-Scans trainiert wird. Die für den RF ausgewählten Merkmale basieren sowohl auf Bildintensitäten sowie auf Kanteninformationen. Des Weiteren werden dem Merkmalsvektor Deskriptoren hinzugefügt, um Umgebungsinformationen zu integrieren. Die Berechnung der Merkmale wird sowohl global als auch lokal in der näheren Nachbarschaft durchgeführt. Im Anschluss an die RF-Segmentierung verwenden wir den graphenbasierten Ansatz aus [3], um die Segmentierung des RF zu optimieren. Für die Bewertung unseres Ansatzes führen wir eine Leave-One-Out-Evaluation durch. Hierbei wird ein mittlerer Dice-Koeffizient von $0.921 \pm 0.045 \mu \mathrm{m}$ und eine mittlere symmetrische Oberflächendistanz von $11.80 \pm 6.05 \mu \mathrm{m}$ erreicht. Darüber hinaus konnte gezeigt werden, dass die graphenbasierte Optimierung eine erhöhte räumliche Konsistenz und Genauigkeit gewährleistet.

\section{Literaturverzeichnis}

1. Manstein D, Laubach H, Watanabe K, et al. Selective cryolysis: a novel method of non-invasive fat removal. Laser Surg Med. 2008;40(9):595-604.

2. Kepp T, Droigk C, Casper M, et al. Segmentation of subcutaneous fat within mouse skin in 3D OCT image data using random forests. In: Proc SPIE; 2018. Accepted.

3. Li K, Wu X, Chen DZ, et al. Optimal surface segmentation in volumetric images a graph-theoretic approach. IEEE Trans Pattern Anal Mach Intell. 2006;28(1):119134. 